\title{
Effect of a Low-Intensity Pulsed Ultrasound Device, SX-1001, on Clinical Symptoms in Buerger Disease With Limb Ischemia
}

\author{
A Multicenter, Double-Blind, Parallel Randomized Clinical Trial
}

\author{
Yukihito Higashi, ${ }^{1,2}$ MD, Nobuyoshi Azuma, ${ }^{3}$ MD, Yasuchika Takeishi, ${ }^{4}$ MD, Tohru Minamino, ${ }^{5}$ MD, \\ Yasuki Kinara, ${ }^{6}$ MD, Koichi Node, ${ }^{7}$ MD, Masataka Sata, ${ }^{8}$ MD, Yoshihiro Fukumoto, ${ }^{9}$ MD, \\ Hideki Origasa, ${ }^{10} \mathrm{PhD}$, Hiroshi Matsuo, ${ }^{11} \mathrm{MD}$, Hiroaki Naritomi, ${ }^{12} \mathrm{MD}$, \\ Masatoshi Fujita, ${ }^{13} \mathrm{MD}$, and Wataru Shimizu, ${ }^{14} \mathrm{MD}$
}

\begin{abstract}
SUMMARY
Buerger disease is a rare disease of unknown etiology and cannot be treated by bypass surgery or percutaneous reendovascularization. Although the need for effective limb ischemia prevention strategies is increasingly being recognized, effective preventative strategies are insufficient. The aim of this study using a new pulsed ultrasound device, SX1001 , is to determine whether treatment using SX-1001 can mitigate rest pain and improve blood supply to ischemic legs in patients with Buerger disease. This study is a multicenter, double-blinded, parallel randomized clinical trial testing the efficacy and safety of SX-1001. Treatment using SX-1001 is expected to result in reduction of the visual analog scale score for pain in Buerger disease patients who have Fontaine stage III. A total of 44 patients from 20 hospitals in Japan will be enrolled. The primary endpoint of the trial is a change in rest pain intensity on the visual analog scale score from baseline to 24 weeks. This trial will be the first to show the safety and efficacy of low-intensity pulsed ultrasound using SX-1001 for clinical symptoms in patients with Buerger disease. Low-intensity pulsed ultrasound may be a new therapy for limb ischemia. Ethical approval has been obtained from each of the participating institutes. Study findings will be disseminated through peer-reviewed journals and at scientific conferences.
\end{abstract}

This study is registered at UMIN Clinical Trial Registry (UMIN000014757). (Int Heart J 2015; 56: 632-638)

Key words: Peripheral arterial disease, Perfusion parameters, Walking performance, Rest pain intensity, Visual analog scale score

$\mathrm{R}$ ecently, the concept of angiogenesis has provided a new option for critical limb ischemia (CLI) treatment. Mechanisms of vascular development and differentiation have been studied, and it has been clarified that angiogenesis is induced by differentiation and proliferation of endothelial progenitor cells (EPCs) or by EPCs mobilized from bone marrow and peripheral blood as well as by proliferation and migration of EPCs from existing blood vessels. ${ }^{1-5)}$ Several investigators have shown the efficacy of gene therapies using vascular growth factors or cell therapies for angiogenesis in patients with CLI. ${ }^{6-13)}$ Unfortunately, both gene and cell therapies are not always satisfactory in patients with peripheral arterial disease (PAD). A more effective and simple way for induc- ing angiogenesis is needed.

Low-intensity pulsed ultrasound irradiation has been widely used to promote the healing of bone fractures in humans. ${ }^{14-16)}$ It is postulated that the initial step of bone fracture healing by low-intensity pulsed ultrasound is angiogenesis, and subsequent neovascularization leads to the migration of osteoblasts to the bone fracture site. Indeed, some studies have shown that low-intensity pulsed ultrasound induces angiogenesis in experimental hind-limb ischemic models. ${ }^{17-19)}$ However, there is no information on the effects of pulsed ultrasound on angiogenesis and clinical symptoms in humans. We hypothesized that low-intensity pulsed ultrasound irradiation has a beneficial effect on limb ischemia and improves clinical symp-

From the ${ }^{1}$ Department of Cardiovascular Regeneration and Medicine, Research Institute for Radiation Biology and Medicine, Hiroshima University, ${ }^{2}$ Division of Regeneration and Medicine, Medical Center for Translational and Clinical Research, Hiroshima University Hospital, Hiroshima, ${ }^{3}$ Department of Vascular Surgery, Asahikawa Medical University, Asahikawa, ${ }^{4}$ Department of Cardiology and Hematology, Fukushima Medical University, Fukushima, ${ }^{5}$ Department of Cardiovascular Biology and Medicine, Niigata University Graduate School of Medical and Dental Sciences, Niigata, ${ }^{6}$ Department of Cardiovascular Medicine, Graduate School of Biomedical and Health Sciences, Hiroshima University, Hiroshima, ${ }^{7}$ Cardiovascular Medicine, Saga University Faculty of Medicine, Saga, ${ }^{8}$ Department of Cardiovascular Medicine, Tokushima University Hospital, Tokushima, ${ }^{9}$ Department of Internal Medicine, Division of Cardio-Vascular Medicine, Kurume University School of Medicine, Kurume, ${ }^{10}$ Division of Biostatistics and Clinical Epidemiology, University of Toyama School of Medicine, Toyama, ${ }^{11}$ Matsuo Vascular Ultrasound Laboratory, Matsuo Clinic, ${ }^{12}$ Senrichuo Hospital, Osaka, ${ }^{13}$ Human Health Sciences, Kyoto University Graduate School of Medicine, Uji Hospital, Uji, and ${ }^{14}$ Department of Cardiovascular Medicine, Nippon Medical School, Tokyo, Japan.

Address for correspondence: Yukihito Higashi, MD, Department of Cardiovascular Regeneration and Medicine, Research Institute for Radiation Biology and Medicine (RIRBM), Hiroshima University, 1-2-3 Kasumi, Minami-ku, Hiroshima 734-8551, Japan. E-mail: yhigashi@hiroshima-u.ac.jp

Received for publication May 8, 2015. Revised and accepted May 21, 2015.

Released in advance online on J-STAGE November 9, 2015.

All rights reserved by the International Heart Journal Association. 
toms by inducing angiogenesis in patients with limb ischemia. Thus, we developed a novel ultrasound device for treatment of limb ischemia. The efficacy of the device for improving clinical symptoms has not yet been tested.

Buerger disease is a rare disease of unknown etiology and cannot be treated by bypass surgery or percutaneous re-endovascularization. Therefore, Buerger disease is a good target for therapeutic angiogenesis. Recently, we have shown that cell therapy with bone-marrow mononuclear cell implantation is safe and effective in patients with CLI, especially in patients with Buerger disease. ${ }^{20)}$ In this trial, we are enrolling Buerger disease patients who have Fontaine stage III.

This trial is a verification study of the effect and safety of pulsed ultrasound for cases of limb ischemia that do not respond to conventional oral administration or surgical treatment or for which conventional treatments are not suitable.

\section{METHODS}

Design: This study is a double-blinded, dummy-controlled parallel-group ultrasound therapy trial using a newly developed device, SX-1001, in Buerger disease patients who have Fontaine stage III. For enrolment, patients must have limb ischemia with ischemic lesions. The eligibility of a patient will

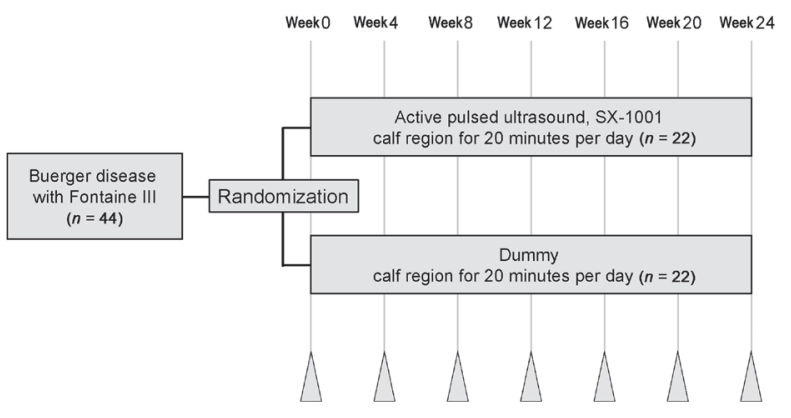

Figure 1. Design schematic of time schedule of the trial. be confirmed by the independent Case Assessment Committee consisting of specialists of vascular surgery, cardiology, radiology, and stroke before starting ultrasound treatment. Standard therapy recommended by Trans Atlantic Inter-Societal Consensus II (TACS II) will be maintained during the entire study period without changing the doses of concomitant medications or physical therapy program. The trial design and schedule are presented in Figure 1 and Table I, respectively. All patients are reviewed prior to study and at $4,8,12,16,20$, and 24 weeks during the treatment phase for assessment of both efficacy and safety.

Study population: Buerger disease will be diagnosed by the previously described criteria, ${ }^{21)}$ including results of physical examinations, clinical symptoms, and angiographic findings: smoking history, onset before the age of 50 years, infrapopliteal arterial occlusive disease, either upper limb involvement or phlebitis migrans, and absence of atherosclerotic risk factors other than smoking. To rule out other vasculitis and hypercoagulable states, rheumatoid factor, lupus anticoagulants, and serologic investigations will be evaluated. The diagnosis of limb ischemia will be confirmed by angiography. Limb ischemia will be classified according to the guidelines of TASC II. ${ }^{22)}$ The target population is patients with Buerger disease in the lower extremity region who have Fontaine classification III. Patients have below the knee disease based on angiographic findings. Patient age will be limited to $\geq 20$ years and $<65$ years. Patients must quit smoking for at least 3 months and be negative for gas chromatography (GC)/mass spectrometry (MS) screening with a plasma cotinine level over $10 \mathrm{ng} /$ $\mathrm{mL}$ during enrolment. A total of 44 patients will be enrolled in this study and they must maintain a constant lifestyle throughout the study. Inclusion criteria are shown in Table II, and exclusion criteria are shown in Table III. The study protocol has been approved by the relevant institutional review boards. Written informed consent for participation in the study will be obtained from all subjects.

Randomization and masking: Eligible patients with Fontaine III are randomly assigned to receive treatment with either active ultrasound equipment (SX-1001, Nippon Sigmax Co., Ltd,

Table I. Trial Schedule

\begin{tabular}{|c|c|c|c|c|c|c|c|}
\hline \multirow{2}{*}{ Parameter } & \multirow{2}{*}{$\begin{array}{l}\text { Screening } \\
\text { until day } 0\end{array}$} & \multicolumn{6}{|c|}{ Treatment period (weeks) } \\
\hline & & 4 & 8 & 12 & 16 & 20 & 24 \\
\hline Informed consent & ○ & & & & & & \\
\hline Enrolment & $\circ$ & & & & & & \\
\hline Clinical characteristics & ○ & & & & & & \\
\hline Smoking context & $\circ$ & $\circ$ & $\circ$ & $\circ$ & $\circ$ & $\circ$ & $\circ$ \\
\hline Smoke-out screening & ○ & ○ & ○ & ○ & & & ○ \\
\hline Systemic hemodynamics & $\circ$ & $\circ$ & $\circ$ & $\circ$ & $\circ$ & $\circ$ & $\circ$ \\
\hline Hematologic test & $\circ$ & ○ & ○ & ० & & & ० \\
\hline Biochemical test & $\circ$ & $\circ$ & ० & $\circ$ & & & ○ \\
\hline Urinary test & $\circ$ & ○ & ० & $\circ$ & & & ○ \\
\hline Pregnancy test & $\circ$ & & & & & & ○ \\
\hline Subjective and objective symptoms & $\circ$ & ○ & ○ & $\circ$ & $\circ$ & ० & ० \\
\hline Visual analog scale & ○ & ○ & ○ & $\circ$ & $\circ$ & $\circ$ & ○ \\
\hline Transcutaneous oxygen pressure & $\circ$ & & & $\circ$ & & & ० \\
\hline Skin perfusion pressure & ○ & & & ○ & & & ○ \\
\hline Ankle brachial pressure index & $\circ$ & & & $\circ$ & & & $\circ$ \\
\hline Toe brachial pressure index & ○ & & & $\circ$ & & & ○ \\
\hline Walking distance & $\circ$ & & & $\circ$ & & & ० \\
\hline Adverse event & & ○ & $\circ$ & ○ & ○ & ○ & ○ \\
\hline
\end{tabular}


Table II. Inclusion Criteria

\begin{tabular}{l} 
Variable \\
\hline Aged 20 years or over and less than 65 years \\
Meeting the diagnosis criteria of the specified and intractable vasculitic disease study group of the Japanese Ministry of \\
Health and Welfare \\
Signed informed consent \\
Limb ischemia of Fontaine stage III \\
Below the knee disease of angiographic findings \\
Non-smoking during the study period confirmed by plasma cotinine level \\
Non-improvement of symptoms of Buerger disease with conventional therapy recommended by Trans-Atlantic Inter-So- \\
ciety Concensus (TASC) II \\
Outpatient visits during the 24 weeks of treatment
\end{tabular}

Table III. Exclusion Criteria

Variable
Planned major amputation during the study period
Possibility of successful by-pass surgery or catheter treatment on the index leg
Applicability of by-pass surgery or catheter treatment on the index leg
Upper knee disease based on angiographic findings
Active malignant disease, severe malfunction of the liver or kidneys and the forefoot evidenced by imaging (radiography)
Not expected to survive more than 12 months, in the opinion of the principal investigator of the study site
A history of malignant disease or previous or present history of cardiac infarction or stroke within 3 months before re-
cruitment
Cardiovascular diseases of New York Heart Association class III or IV or active angina
Current or planned pregnancy during the study period
Previous treatment in another investigational clinical trial within 6 months before recruitment
Heavy second-hand tobacco smoke or patients excluded based on the opinion of the principal investigator of the study site

Tokyo) or dummy equipment (ratio 1:1). The random code list is electrically generated by an independent trial statistician where the clinical center is assigned to a block. Randomization is managed by a third-party enrolment center.

Procedures: Subjects will be given detailed instructions and a manual for operation of the equipment and instructed to use the equipment on the index leg daily at home 20 minutes every day for 24 weeks. Operation records including starting and completion times will be automatically recorded by the logger in the equipment. Subjects will be advised to visit the outpatient clinic once every 4 weeks. Ultrasound irradiation will be applied over the skin of the ischemic area of the calf region for 20 minutes per day during the 24 -week period. The gap between the transducer and skin will be filled with ultrasonic gel. A transducer (Nippon Sigmax Co., Ltd, Tokyo) generates a pulsed $2.0-\mathrm{MHz}$ signal with a $200-\mu$ second pulse burst and $1-\mathrm{kHz}$ repetition rate at the intensity of $30 \mathrm{~mW} / \mathrm{cm}^{2}$ and a pulse wave with a duty cycle of $20 \%$. In this trial, 8 transducers will be attached to the skin over the gastrocnemius of each ischemic leg (Figure 2). If the disease has affected both legs and both are unsuitable for re-vascularization, the leg with the lower ankle brachial pressure index (ABI) will be identified as the leg to be treated (index leg). The adverse effects and worsening symptoms for the other leg will be recorded in the case report form.

Endpoints: Primary and secondary endpoints are shown in Table IV. The primary endpoint of the trial is the change in rest pain intensity on a visual analog scale (VAS) from baseline to 24 weeks. The secondary endpoints are the change in rest pain intensity on the VAS from baseline to 12 weeks, changes in transcutaneous oxygen pressure $\left(\mathrm{TcPO}_{2}\right)$, skin perfusion pressure (SPP), ABI, toe brachial pressure index (TBI), and walking distance from baseline to 12 and 24 weeks, and changes in symptoms related to Buerger disease in the study period of 24 weeks.

\section{Measurements:}

Primary endpoint Rest pain intensity is assessed by a self-administered VAS from baseline to 24 weeks as previously described. ${ }^{23-25)}$ Briefly, observers place the VAS line at a point that represents the pain intensity of respondents. The respondents complete the VAS line presentation by themselves within 1 minute. The VAS score is a horizontal line of $10 \mathrm{~cm}$ in length, with $0 \mathrm{~cm}$ corresponding to no pain and $10 \mathrm{~cm}$ corresponding to most severe pain. The observers are blind to the form of ex- 

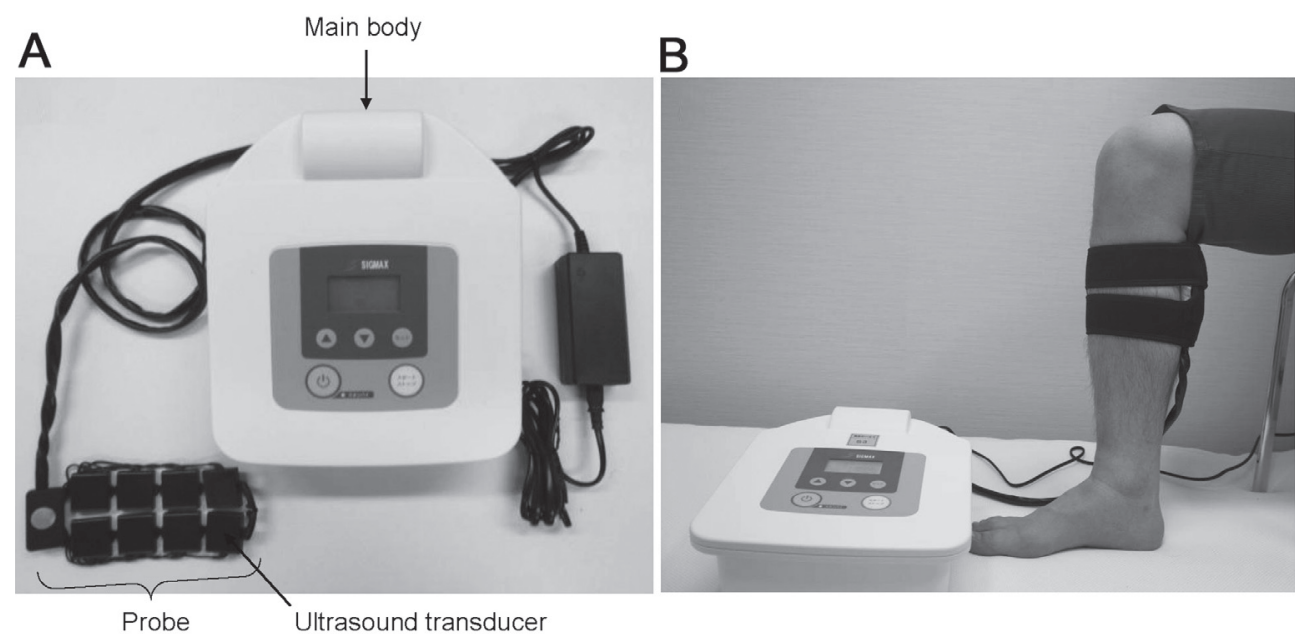

Figure 2. A: Appearance of the low-intensity pulsed ultrasound device. B: Application of transducers for LIPUS irradiation in the gastrocnemius of the ischemic leg.

Table IV. Endpoints

\begin{tabular}{ll}
\hline Variable & \\
\hline Primary endpoint & . Change in rest pain intensity on a visual analog scale (VAS) from baseline to 24 weeks \\
Main secondary endpoints & $\cdot$ Change in rest pain intensity on a VAS from baseline to 12 weeks \\
& $\cdot$ Changes in transcutaneous oxygen pressure (TcPO $)$, skin perfusion pressure (SPP), ankle \\
& brachial pressure index (ABI), toe brachial pressure index (TBI) and walking distance from \\
baseline to 12 and 24 weeks & \\
& Changes in symptoms related to Buerger disease in the study period of 24 weeks
\end{tabular}

amination.

Secondary endpoints

1) Rest pain: Rest pain intensity is assessed by a self-administered VAS from baseline to 12 weeks.

2) Perfusion parameters: All of the studies will be performed in the morning, after overnight fasting, in a quiet, dark and airconditioned room (constant temperature of $22-25^{\circ} \mathrm{C}$ ). The subjects will remain in a supine position throughout the study. Perfusion parameters are measured after a 15 -minute rest period. $\mathrm{TcPO}_{2}$ will be measured by an electrochemical transducer (TCM 400, Radiometer K.K., Tokyo) as previously described. ${ }^{26,27)}$ Briefly, skin at the measured site will be shaved and cleaned with alcohol to ensure optimal $\mathrm{TcPO}_{2}$ electrode contact for skin oxygen diffusion. The $\mathrm{TcPO}_{2}$ electrodes stabilized at $43^{\circ} \mathrm{C}$ will then be placed on the skin at the measured site. $\mathrm{TcPO}_{2}$ values will be recorded after a 20-minute stabilization period for the electrodes. SPP will be measured by a SensiLase PAD3000 flowmeter (Kaneka Medix Co., Osaka, Japan) as previously described. ${ }^{28)}$ Briefly, a laser Doppler probe positioned within the blood pressure cuff will be wrapped around the subject's foot. ABI and TBI will be measured with a new device (Form PWV/ABI; Omron Colin, Co., Tokyo) as previously described. ${ }^{29,30)}$ Briefly, the subjects will be kept in the supine position for at least 5 minutes and cuffs will be wrapped around both brachia and ankles. An oscillometric method is used to measure systolic blood pressure in the bilateral brachial and posterior tibial arteries. The ABI value is calculated by dividing the highest pressure in the posterior tibial arteries on the right and left sides by the highest brachial pressure on either side. To measure toe pressure, appropriately sized cuffs will be placed at the base of the toe. The TBI value is calculated by dividing the highest pressure in the toe on the right and left sides by the highest brachial pressure on either side. All procedures will be performed in accordance with the manufacturer's protocol. The observers will be blind to the form of examination.

3) Walking performance: Maximal walking distance/time or pain-free walking distance/time or both will be measured to evaluate walking performance by a treadmill test under the conditions of $2.4 \mathrm{~km} / \mathrm{h}$ and $12 \%$ degrees in incline of the treadmill device, and the maximum walking distance will be measured up to $400 \mathrm{~m}$ during a period of 10 minutes.

\section{Others}

1) Clinical characteristics: Before starting exposure, the following will be checked and recorded: primary doctor, medical record number, patient's initial, sex, birth date, height, body weight, diagnosed disease, risk factors for coronary artery disease, smoking history, family history, treatment upon enrolment, in-house or out-patient, and concomitant diseases.

2) Smoking-out screening test: Smoking-out is confirmed by a negative result for GC/MS screening with a plasma conitine level over $10 \mathrm{ng} / \mathrm{mL}$ during enrolment.

3) Systemic haemodynamics: Systolic pressure, diastolic pressure, and heart rate will be measured by the standard sphygmomanometric method in each treatment period.

4) Hematologic and biochemical tests : Baseline fasting levels 
of hematocrit, hemoglobin, red blood cells, white blood cells, white blood cell fraction, and platelets and fasting serum concentrations of aspartate aminotransferase, alanine aminotransferase, bilirubin, alkaline phosphatase, gamma-guanosine triphosphate, creatine kinase, serum protein, serum albumin, C-reactive protein, total cholesterol, triglycerides, low-density lipoprotein cholesterol, high-density lipoprotein cholesterol, glucose, and $\mathrm{HbA} 1 \mathrm{c}$ will be measured.

5) Urinary test: Baseline fasting urinary concentrations of urinary protein, albumin, and creatinine will be measured.

6) Pregnancy test: Before starting exposure, a pregnancy test using measurement of urinary excretion of human chorionic gonadotropin will be performed in pre-menopausal women.

7) Adverse effects : Abnormal laboratory data will be included in adverse effects. The primary doctor will give necessary treatment and fully evaluate the follow-up process. The primary doctor will record adverse effect details, date of onset and/or disappearance, degree, treatment, outcome, severity, and causal relationship to the low-intensity ultrasound exposure in the medical record. Except for unrelated adverse effects, the patient will be followed up as long as possible until recovery. Safety assessments will include all adverse and serious adverse events, subjective symptoms, and blood tests. A history of cancer or an active tumor will be an exclusion criterion (Table III). Equipment: Figure 2A shows the newly developed pulsed ultrasound device, SX-1001.

Specifications

1) Size: $249 \mathrm{~mm}(\mathrm{~W}) \times 298 \mathrm{~mm}(\mathrm{D}) \times 142 \mathrm{~mm}(\mathrm{H})$

2) Weight: $1.9 \mathrm{~kg}$

3) Power: $\mathrm{AC} 100 \mathrm{~V} \pm 10 \%$

4) Apparent power: $35 \mathrm{VA}$ or less

5) Protection Class for electroconvulsion: Class II

6) Protection Type for electroconvulsion: Type BF

7) Ultrasound specifications:

- Channels: 8 channels

- Acoustic Frequency: $2 \mathrm{MHz} \pm 10 \%$

- Intensity (Isata): $30 \mathrm{~mW} / \mathrm{cm}^{2} \pm 10 \%$

- Pulse Repetition Frequency (PRF): $1 \mathrm{msec} \pm 5 \%(1 \mathrm{kHz} \pm$ $5 \%)$

- Duty: $20 \pm 5 \%$

Dummy equipment Dummy equipment is the same size and weight as that of the SX-1001. It operates in the same way as the SX-1001. The dummy shows the same display as that of SX-1001 during operation and cannot be distinguished from the active equipment by the study subjects or the investigator.

Compliance After treatment of 20 minutes, both devices stop operating automatically. A daily record of operation starting and completion times will be stored in the logger in the equipment during the entire study period for assessment of treatment compliance.

Application of transducers Figure 2B shows the application of transducers for low-intensity pulsed ultrasound irradiation in the gastrocnemius of the ischemic leg.

Data analysis:

Sample size estimation This study hypothesized that the SX1001 would reduce the rest pain score as compared to the dummy instrument during the 24 weeks of the treatment period. The difference in the decrease of the pain score during the 24 weeks between the two groups was assumed to be 1.0 with a standard deviation of 1.1 based on a preliminary study. To detect such a difference statistically, 22 patients per group will be required to achieve $80 \%$ power assuming an attrition of $10 \%$.

Statistical analysis plan Mean difference in the absolute change of the rest pain score during the 24 weeks will be compared between the two groups by the analysis of covariance with a baseline score as a covariate. A missing value of the rest pain score at 24 weeks will be imputed according to the principle of the last observation carried forward. A mixed-effects model will be also applied to the repeated measured data as a sensitivity analysis. Similar analyses will be applied to the secondary endpoints of $\mathrm{TcPO}_{2}$, SPP, TBI, and walking distance. Foot ulcer elimination rates will be compared between the two groups using Fisher's exact test. Statistical significance will be assessed at a two-sided 5\% level.

\section{Discussion}

This trial will be the first study showing the safety and efficacy of low-intensity pulsed ultrasound for clinical symptoms in patients with Buerger disease using a randomized, doubleblinded, and placebo-controlled design. Low-intensity pulsed ultrasound may be a new therapy for limb ischemia.

In a clinical setting, low-intensity pulsed ultrasound irradiation has been widely used to promote the healing of bone fractures. ${ }^{14-16)}$ Angiogenesis should be the initial step of bone fracture healing by low-intensity pulsed ultrasound, and subsequent neovascularization leads to the migration of osteoblasts to the bone fracture site. Although there is no information on the effects of low-intensity pulsed ultrasound on angiogenesis in humans, a few studies have shown that low-intensity pulsed ultrasound induces angiogenesis in experimental hind-limb ischemic models. ${ }^{17-19)}$ It is expected that low-intensity pulsed ultrasound irradiation will improve clinical symptoms by inducing angiogenesis in patients with Buerger disease.

Ultrasound induces an increase in shear stress on the surface of endothelial cells. ${ }^{31)}$ It is well known that acute or chronic increases in shear stress patently stimulate the release of nitric oxide (NO) in isolated vessels and cultured cells. ${ }^{32,33)}$ Therefore, ultrasound also may act as a mechanical stimulator of NO release. Indeed, Suchkova, et $a l^{18)}$ have shown that vasodilatory effects of low-intensity pulsed ultrasound are completely abolished by an NO synthase inhibitor in a rabbit hindlimb ischemic model. This beneficial effect of low-intensity pulsed ultrasound irradiation may also be expected in patients with PAD.

It is thought that low-intensity pulsed ultrasound, in vitro and in vivo, induces angiogenesis through increases in angiogenic growth factors and angiogenic cytokines such as interleukin-8, basic fibroblast growth factor, and vascular endothelial growth factor. ${ }^{34,35)}$ Angiogenic transcription factors, angiogenic cytokines and their receptors would contribute to ultrasound-induced angiogenesis. The therapeutic range of ultrasound stimulates both NO and prostaglandin E2 synthesis in human osteoblasts. ${ }^{36)}$ Exposure of endothelial cells to therapeutic ultrasound increases NO synthase activity and NO production, which could lead experimentally or therapeutically to the induction of vasodilatation. ${ }^{37)}$ Ultrasound irradiation of 1.6$2.0 \mathrm{~W} / \mathrm{cm}^{2}$ in intensity for 6 days enhanced $\mathrm{NO}$ and $\mathrm{Ca}^{2+}$ release from endothelial cells but did not promote endothelial cell growth. ${ }^{38)}$ In addition, ultrasound irradiation altered cellu- 
lar morphology and orientation and increased extracellular matrix secretion from endothelial cells. ${ }^{16)}$ It is expected that a benign circle among angiogenesis, improvement of endothelial function, and increase in NO promotes more effective angiogenesis during low-intensity pulsed ultrasound irradiation, leading to improvement of clinical symptoms.

Study limitations: There are a number of limitations concerning assessment of pain using a VAS. In a large number of trials for assessment of chronic pain, such as rheumatoid arthritis and osteoarthritis, VAS is used as the primary endpoint. ${ }^{39,40)} \mathrm{A}$ good agreement between VAS score and patient-reported pain reduction has been shown in patients with cancer-related pain. ${ }^{41)}$ However, there is no internationally agreed upon definition of pain scale in patients with PAD. In our trial, we selected VAS as the primary endpoint since the subjects are patients with Buerger disease who have Fontaine III. We believe that a visual pain scale is a suitable endpoint in this trial. It has been reported that a clinical improvement in pain using the Global Rating Scale relates to a $30 \%$ reduction in pain. ${ }^{42}$ Rosier, et $\mathrm{l}^{43)}$ have shown that repeated VAS measurements have a variability of approximately $20 \%$ when evaluating reproducibility of pain measurement using a VAS. It is thought that reduction in VAS of $30 \%$ means a significant decrease in pain status. In a preliminary study, we confirmed that low-intensity pulsed ultrasound decreased VAS from $6.4 \pm 0.8(\mathrm{SD})$ to $4.1 \pm$ 0.6 (SD) $(P<0.01)$ in 12 patients with Buerger disease who have Fontaine III and IV. However, unfortunately, since there is no golden standard for validity of pain criteria at present, we do not know what a change of 1 in VAS score actually means, especially in patients with PAD. Further studies are needed to develop and validate a standardized VAS measurement.

In conclusion, low-intensity pulsed ultrasound may be a new strategy for improving ischemic limb symptoms. If lowintensity pulsed ultrasound is shown in this trial to be effective for improving clinical symptoms in patients with Buerger disease who have limb ischemia, further studies will be needed to confirm the efficacy of low-intensity pulsed ultrasound for improving clinical symptoms in atherosclerotic patients with PAD and in patients with mild Buerger disease and severe Buerger disease with ulcers and gangrene. In addition, it is expected that low-intensity pulsed ultrasound irradiation to the heart, kidney and liver will be useful for improvement of tissue injury and clinical symptoms in patients with coronary heart disease, kidney disease, and liver disease. The door will be open to expand ultrasound beyond the field of diagnostic imaging.

\section{REFERENCES}

1. Rehman J, Li J, Orschell CM, March KL. Peripheral blood "endothelial progenitor cells" are derived from monocyte/macrophages and secrete angiogenic growth factors. Circulation 2003; 107: 1164-9.

2. Kinnaird T, Stabile E, Burnett MS, et al. Marrow-derived stromal cells express genes encoding a broad spectrum of arteriogenic cytokines and promote in vitro and in vivo arteriogenesis through paracrine mechanisms. Circ Res 2004; 94: 678-85.

3. Ziegelhoeffer T, Fernandez B, Kostin S, et al. Bone marrow-derived cells do not incorporate into the adult growing vasculature. Circ Res 2004; 94: 230-8.

4. Silva GV, Litovsky S, Assad JA, et al. Mesenchymal stem cells differentiate into an endothelial phenotype, enhance vascular density, and improve heart function in a canine chronic ischemia model. Circulation 2005; 111: 150-6.

5. O'Neill TJ 4th, Wamhoff BR, Owens GK, Skalak TC. Mobilization of bone marrow-derived cells enhances the angiogenic response to hypoxia without transdifferentiation into endothelial cells. Circ Res 2005; 97: 1027-35.

6. Tateishi-Yuyama E, Matsubara H, Murohara T, et al; Therapeutic Angiogenesis using Cell Transplantation (TACT) Study Investigators. Therapeutic angiogenesis for patients with limb ischaemia by autologous transplantation of bone-marrow cells: a pilot study and a randomized controlled trial. Lancet 2002; 360: 427-35.

7. Durdu S, Akar AR, Arat M, Sancak T, Eren NT, Ozyurda U. Autologous bone-marrow mononuclear cell implantation for patients with Rutherford grade II-III thromboangiitis obliterans. J Vasc Surg 2006; 44: 732-9.

8. Motukuru V, Suresh KR, Vivekanand V, Raj S, Girija KR. Therapeutic angiogenesis in Buerger's disease (thromboangiitis obliterans) patients with critical limb ischemia by autologous transplantation of bone marrow mononuclear cells. J Vasc Surg 2008; 48: 53S-60S.

9. Higashi Y, Kimura M, Hara K, et al. Autologous bone-marrow mononuclear cell implantation improves endothelium-dependent vasodilation in patients with limb ischemia. Circulation 2004; 109: 1215-8.

10. Miyamoto K, Nishigami K, Nagaya N, et al. Unblinded pilot study of autologous transplantation of bone marrow mononuclear cells in patients with thromboangiitis obliterans. Circulation 2006; 114: $2679-84$

11. Higashi Y, Miyazaki M, Goto C, Sanada H, Sueda T, Chayama K. Sarpogrelate, a selective 5-hydroxytryptamine (2A) antagonist, augments autologous bone marrow mononuclear cell implantation-induced improvement in endothelium-dependent vasodilation in patients with critical limb ischemia. J Cardiovasc Pharmacol 2010; 55: 56-61.

12. Lederman RJ, Mendelsohn FO, Anderson RD, et al; TRAFFIC Investigators. Therapeutic angiogenesis with recombinant fibroblast growth factor-2 for intermittent claudication (the TRAFFIC study): a randomised trial. Lancet 2002; 359: 2053-8.

13. Rajagopalan S, Mohler ER 3rd, Lederman RJ, et al. Regional angiogenesis with vascular endothelial growth factor in peripheral arterial disease: a phase II randomized, double-blind, controlled study of adenoviral delivery of vascular endothelial growth factor 121 in patients with disabling intermittent claudication. Circulation 2003; 108: 1933-8.

14. Xavier CAM, Duarte LR. Estimulacao ultra-sonic de calo osseo: Appicacao clinia. Rev Bras Ortop 1983; 18: 73-80.

15. Heckman JD, Ryaby JP, McCabe J, Frey JJ, Kilcoyne RF. Acceleration of tibial fracture-healing by non-invasive, low-intensity pulsed ultrasound. J Bone Joint Surg Am 1994; 76: 26-34.

16. Kristiansen TK, Ryaby JP, McCabe J, Frey JJ, Roe LR. Accelerated healing of distal radial fractures with the use of specific, lowintensity ultrasound. A multicenter, prospective, randomized, double-blind, placebo-controlled study. J Bone Joint Surg Am 1997; 79: 961-73.

17. Young SR, Dyson M. The effect of therapeutic ultrasound on angiogenesis. Ultrasound Med Biol 1990; 16: 261-9.

18. Suchkova VN, Baggs RB, Sahni SK, Francis CW. Ultrasound improves tissue perfusion in ischemic tissue through a nitric oxide dependent mechanism. Thromb Haemost 2002; 88: 865-70.

19. Barzelai S, Sharabani-Yosef O, Holbova R, et al. Low-intensity ultrasound induces angiogenesis in rat hind-limb ischemia. Ultrasound Med Biol 2006; 32: 139-45.

20. Idei N, Soga J, Hata T, et al. Autologous bone-marrow mononuclear cell implantation reduces long-term major amputation risk in patients with critical limb ischemia: a comparison of atherosclerotic peripheral arterial disease and Buerger disease. Circ Cardiovasc Interv 2011; 4: 15-25.

21. Shionoya S. Diagnostic criteria of Buerger's disease. Int J Cardiol 1998; 66: S243-5. 
22. Norgren L, Hiatt WR, Dormandy JA, et al. Inter-Society Consensus for the Management of Peripheral Arterial Disease (TASC II). Eur J Vasc Endovasc Surg 2007; 33: S1-75.

23. Scott J, Huskisson EC. Accuracy of subjective measurements made with or without previous scores: an important source of error in serial measurement of subjective states. Ann Rheum Dis 1979; 38: 558-9.

24. Huskisson EC. Visual analogue scales. In: Melzack R. Pain measurement and assessment. New York, NY: Raven Press; 1998: 33-7.

25. Morishita R, Aoki M, Hashiya N, et al. Safety evaluation of clinical gene therapy using hepatocyte growth factor to treat peripheral arterial disease. Hypertension 2004; 44: 203-9.

26. de Meijer VE, Van't Sant HP, Spronk S, Kusters FJ, den Hoed PT. Reference value of transcutaneous oxygen measurement in diabetic patients compared with nondiabetic patients. J Vasc Surg 2008; 48: 382-8.

27. Hotta K, Kamiya K, Shimizu R, et al. Stretching exercises enhance vascular endothelial function and improve peripheral circulation in patients with acute myocardial infarction. Int Heart J 2013; 54: 59-63.

28. Castronuovo JJ Jr, Adera HM, Smiell JM, Price RM. Skin perfusion pressure measurement is valuable in the diagnosis of critical limb ischemia. J Vasc Surg 1997; 26: 629-37.

29. Kajikawa M, Maruhashi T, Iwamoto Y, et al. Borderline anklebrachial index value of 0.91 to 0.99 is associated with endothelial dysfunction. Circ J 2014; 78: 1740-5.

30. Ishisone T, Koeda Y, Tanaka F, Sato K, Nagano M, Nakamura M. Comparison of utility of arterial stiffness parameters for predicting cardiovascular events in the general population. Int Heart J 2013; 54: 160-5.

31. Hadjiargyrou M, McLeod K, Ryaby JP, Rubin C. Enhancement of fracture healing by low intensity ultrasound. Clin Orthop Relat Res 1998; 355: S216-29. (Review)

32. Miller VM, Vanhoutte PM. Enhanced release of endothelium-derived factors by chronic increases in blood flow. Am J Physiol
1988; 255: H446-51.

33. Uematsu M, Ohara Y, Navas JP, et al. Regulation of endothelial cell nitric oxide synthase m RNA expression by shear stress. Am J Physiol 1995; 269: C1371-8.

34. Alter A, Rozenszajn LA, Miller HI, Rosenschein U. Ultrasound inhibits the adhesion and migration of smooth muscle cells in vitro. Ultrasound Med Biol 1998; 24: 711-21.

35. Reher P, Doan N, Bradnock B, Meghji S, Harris M. Effect of ultrasound on the production of IL-8, basic FGF and VEGF. Cytokine 1999; 11: 416-23.

36. Reher P, Harris M, Whiteman M, Hai HK, Meghji S. Ultrasound stimulates nitric oxide and prostaglandin $\mathrm{E} 2$ production by human osteoblasts. Bone 2002; 31: 236-41.

37. Altland OD, Dalecki D, Suchkova VN, Francis CW. Low-intensity ultrasound increases endothelial cell nitric oxide synthase activity and nitric oxide synthesis. J Thromb Haemost 2004; 2: 637-43.

38. Hsu SH, Huang TB. Bioeffect of ultrasound on endothelial cells in vitro. Biomol Eng 2004; 21: 99-104.

39. Hewlett S, Hehir M, Kirwan JR. Measuring fatigue in rheumatoid arthritis: a systematic review of scales in use. Arthritis Rheum 2007; 57: 429-39. (Review)

40. Veenhof C, Bijlsma JW, van den Ende CH, van Dijk GM, Pisters MF, Dekker J. Psychometric evaluation of osteoarthritis questionnaires: a systematic review of the literature. Arthritis Rheum 2006; 55: 480-92. (Review)

41. Cepeda MS, Africano JM, Polo R, Alcala R, Carr DB. Agreement between percentage pain reductions calculated from numeric rating scores of pain intensity and those reported by patients with acute or cancer pain. Pain 2003; 106: 439-42.

42. Farrar JT, Young JP Jr, LaMoreaux L, Werth JL, Poole RM. Clinical importance of changes in chronic pain intensity measured on an 11-point numerical pain rating scale. Pain 2001; 94: 149-58.

43. Rosier EM, Iadarola MJ, Coghill RC. Reproducibility of pain measurement and pain perception. Pain 2002; 98: 205-16. 